\title{
Strategic influences on implementing instructions for future actions
}

\author{
Dorit Wenke · Robert Gaschler · Dieter Nattkemper • \\ Peter A. Frensch
}

Received: 9 October 2008 / Accepted: 19 December 2008 / Published online: 10 April 2009

(C) The Author(s) 2009. This article is published with open access at Springerlink.com

\begin{abstract}
Temporal and strategic factors that might influence the transformation of verbal task rules into functional stimulus-response associations were investigated in three experiments. In a dual task paradigm of the ABBA type participants were presented new $\mathrm{S}-\mathrm{R}$ instructions for the A-task at the beginning of each trial. On varying proportions of trials No-go signals rendered the instructed A-task mappings irrelevant before instruction implementation was assessed during performance of an unrelated B-task. Our results indicate that participants refrain from implementing the mappings during instruction presentation when No-go signals appear frequently and late (Exp. 2), and that they can interrupt implementing instructed S-R mappings when frequent No-go signals appear early enough during implementation (Exp. 3). When No-go signals are rare and late, however (Exp. 1), the instructed stimulus features always activate their associated responses during performance of the embedded B-task in an automatic manner. Together, these findings suggest that participants strategically control whether or not they implement verbal instructions. Once implemented, however, instructed S-R associations influence behaviour even when the instructed mappings are no longer task relevant.
\end{abstract}

D. Wenke $(\bowtie)$

Department of Psychology,

Max Planck Institute for Human Cognitive and Brain Sciences,

Stephanstrasse 1a, 04103 Leipzig, Germany

e-mail: wenke@cbs.mpg.de

R. Gaschler · D. Nattkemper · P. A. Frensch

Department of Psychology,

Humboldt University, Berlin, Germany

\section{Introduction}

Unlike other animals, humans with mature and intact frontal cortices can use verbal instructions to acquire new and arbitrary behavioural options seemingly effortlessly and very rapidly. Although the ability to link just about any verbally instructed stimulus feature with just about any instructed motor behaviour seems to be a cornerstone of human cognitive flexibility (Luria, 1961), only a handful of studies (Brass, Wenke, Spengler, \& Waszak, 2009; CohenKdoshay \& Meiran, 2007, 2009; De Houwer, Beckers, Vandorpe, \& Custers, 2005; Waszak, Wenke, \& Brass, 2008; Wenke, Nattkemper, \& Gaschler, 2007) has investigated the functional and neuronal mechanisms that allow humans to immediately behave as instructed.

Some of these experiments show that instructions alone can suffice to create links between instructed stimulus and response features that lead to automatic stimulus-response activation once a stimulus that meets the specified conditions is encountered for the first time (Cohen-Kdoshay \& Meiran, 2007, 2009; De Houwer et al., 2005; Wenke et al., 2007). For example, Wenke et al. instructed a new set of S-R mappings (e.g., N, left key; K, right key) for a future choice task at the beginning of each trial. Before the imperative stimulus for the choice task, the letter identity task, appeared, participants performed an embedded, but logically independent size task. The embedded size task involved two adjacently presented letter stimuli differing in font size (e.g., the letters $\mathrm{N}$ and $\mathrm{K}$, or the letters $\mathrm{F}$ and $\mathrm{B}$ ). Participants judged, by making non-spatial responses, whether the bigger font letter appeared on the left or the right. The instructed letter task mappings influenced size task performance, although they were useless for performing the size task. Specifically, size task responses were slower when the positions of the letters did not correspond 
to the instructed response locations (incompatible condition; e.g., the letters $\mathrm{N}$ and $\mathrm{K}$ appeared at right and left positions, respectively) than in conditions in which (a) overlapping letters appeared at the corresponding positions (compatible condition; e.g., the letter $\mathrm{N}$ appeared on the left and the letter $\mathrm{K}$ appeared on the right), or (b) non-overlapping letters served as size task stimuli (neutral condition; e.g., the letters $\mathrm{F}$ and $\mathrm{B}$ ). These findings suggest that instructions were used to create bindings between the specified stimulus features (letter identity) and response features (response location). The resulting links became automatically activated when participants encountered the same letter stimuli in the logically independent size task, hence delaying size task reactions when the letters appeared at screen positions that did not correspond to the instructed response locations.

These results (also see Cohen-Kdoshay \& Meiran, 2007; Colgan, 1970; Cook \& Harris, 1937; De Houwer et al., 2005) suggest that instructions can be used to control behaviour in a prepared reflex-like manner (Exner, 1879; Gollwitzer, 1999; Hommel, 2000; Woodworth, 1938). The prepared reflex metaphor holds that intentional control is needed for implementing the required cognitive control structures or task sets (Cohen-Kdoshay \& Meiran, 2007; Hommel, 2000; Kunde, Kiesel, \& Hoffmann, 2003; Woodworth, 1938). Once implemented, however, external stimuli that meet the specified environmental conditions automatically trigger the required action without further need of effortful or intentional "translation".

Thus far, the majority of studies on instruction implementation have focussed on automatic S-R translation once the instructed mappings are implemented. In contrast, the allegedly controlled process of implementation itself has received little attention. The present experiments target the supposedly controlled implementation of instructions that precedes automatic $\mathrm{S}-\mathrm{R}$ translation when performing the instructed task. We explore to what extent strategic and temporal factors influence whether or not verbal task rules are implemented in advance. Specifically, we investigate whether, and under which conditions, participants transform verbal $\mathrm{S}-\mathrm{R}$ instruction into functional associations even when instructions are cancelled, thereby rendering the instructed S-R mappings completely irrelevant for future behaviour.

Cancelling or avoiding instruction implementation can be assumed to be a crucial cognitive control ability that is important in everyday life. Imagine, for example, that you need to ask somebody for directions in a foreign city. This somebody might recognise an error in the direction he/ she provides, and ask you to just forget about part of the instructions. Certainly it would be most efficient to either not encode these erroneous instructions at all or to delete them from the already established sub-linguistic representation of the instructed route. Otherwise you would "automatically" get lost. However, is it possible to abort implementation of these instructions? Assuming that instruction implementation is indeed a controlled process, one might expect that it is possible to strategically decide on whether or not to implement the instructions online, depending on how reliable the informant is. While it may be the most efficient strategy in terms of time and error likelihood to implement the instructions online when instructions are likely to be valid, it would be better to adopt a "wait and see" strategy if they are not (Hourihan $\&$ Taylor, 2006). Another factor that might influence the likelihood of advance implementation of instructions for future behaviour (given by a reliable informant) might be the time at which our source renders the instructions invalid. Again assuming flexible cognitive control over instruction implementation it should be easier to abort implementation when the No-go signal comes early than when it arrives after instructions have already been implemented to some extent.

We tested these predictions adopting the paradigm developed by Wenke et al. (2007; see Fig. 1, and description above). As in the Wenke et al. (2007) study, new twochoice mapping instructions assigning specific letters to left and right responses for the letter identity task were given in each trial and were followed by an intermittent size task that required judging where the physically larger of two adjacently presented letters appeared.

The purpose of the size task was to assess the impact of not yet performed identity task instructions: cross-task compatibility effects in the size task served as a measure of automatic S-R activation. As before, we expected a crosstask compatibility effect to show up if letter task instructions are implemented in advance while they are read.

The crucial manipulation in the current experiments involved No-go signals that rendered the instructed letter task mappings completely irrelevant. On No-go trials, the mapping instructions for the letter identity task turned red during instruction presentation (see Fig. 1). A colour change signalled that participants must not respond to the letter identity task target presented at the end of each trial. The proportion of No-go trials was either low (Exps. 1 and 3) or high (Exp. 2). Moreover, No-go signals appeared either late during instruction presentation (Exps. 1 and 2), or they were presented early on (Exp. 3).

Critically, the No-go signals only referred to the letter identity task. In contrast, the embedded size task had to be performed on both, identity task Go trials and identity task No-go trials. This allowed us to directly assess strategic control over instruction implementation: if reading task instructions induced functional associations between instructed stimulus and response features, then we should observe similar cross-task compatibility effects between the 
stimulus positions of the letters in the size task and the response locations assigned to these letters during letter task instructions as those observed earlier (Wenke et al., 2007). That is, we should find evidence for automatic stimulus-response activation during performance of the size task, regardless of whether or not the instructions are still valid for later identity task performance. In contrast, if participants can decide to interrupt verbal rule implementation on No-go trials, or refrain from implementing verbal rules to start with, then the compatibility effect should be reduced or absent. We predicted that participants would be more likely to successfully counteract preparation when No-go signals appeared early (Exp. 3) or frequently (Exp. 2) compared to when No-go signals appeared rarely and late during instruction presentation (Exp. 1).

\section{Experiment 1}

In the first Experiment, No-go signals appeared late during instruction presentation (see Fig. 1), allowing relatively much time for implementation, and relatively little time for cancelling implementation of the instructed mappings. Moreover, the ratio of Go to No-go trials was 2:1, so that participants presumably expected a given trial to be a Go trial.

We assumed that, under these conditions, participants would at least begin to implement the instructed S-R mappings on No-go trials. The impact of instruction implementation on size task performance on No-go trials should depend on how flexibly participants can interrupt and cancel the implementation process. On the one hand, it is possible that participants can interrupt preparation and delete the instructed mappings from working memory at any time during instruction implementation. This view seems consistent with findings suggesting that instructioninduced bindings are kept in some sort of working memory (Cohen-Kdoshay \& Meiran, 2007) and may be rather weak compared to $\mathrm{S}-\mathrm{R}$ associations consolidated by practice (Waszak et al., 2008; Brass et al., 2009; see "General discussion" for details). If participants can cancel instruction implementation at any point during preparation, we should not observe a cross-task compatibility effect in size task responses on No-go trials.

On the other hand, it is also conceivable that instruction implementation has proceeded too far to be successfully aborted when a late No-go signal finally appears. Accordingly, one might expect the instructed mappings to influence
Fig. 1 Schematic illustration of a cross-task incompatible trial (left panel), a non-overlapping neutral trial (middle panel), and a compatible trial (right panel). Trials started with the instruction for the identity task. No-go trials (identity task only) were signalled by colouring the instructions red for the last $300 \mathrm{~ms}$ (Exps. 1 and 2) or $2,600 \mathrm{~ms}$ of the $3,000 \mathrm{~ms}$ instruction duration (Exp. 3). After the size task response, participants either responded to the imperative stimulus of the identity task as instructed (Go trials), or they had to withhold responding (No-go and catch trials)

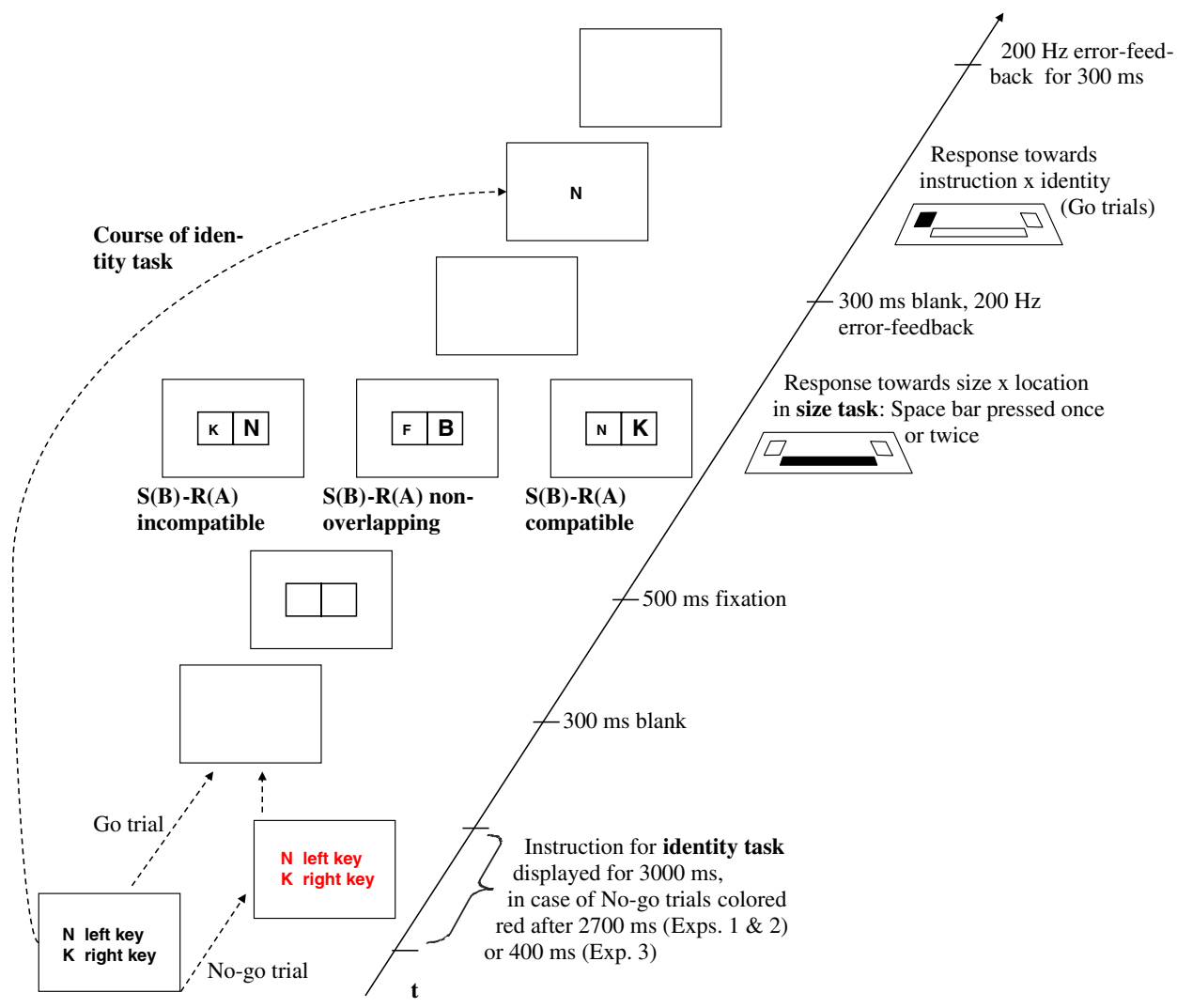


size task performance although the No-go signal rendered the instructed mappings useless.

Finally, we hypothesized that participants complete advance implementation of the mapping instructions on Go trials, and hence predicted a cross-task compatibility effect in size task responses on Go trials.

\section{Methods}

\section{Participants}

Thirty students from the city of Berlin ( 20 female, mean age $=24.4$ years) received $€ 12$ or partial course credit for participation. All participants had normal or corrected to normal vision.

\section{Design and procedure}

Design and procedure were based on Wenke et al. (2007) with the only difference that the Go/No-go manipulation was added. New pairs of S-R mappings for the identity task were instructed at the beginning of each trial (e.g., N: left key, K: right key, on trial $n$; and D: left key, Z: right key, on trial $n+1$; see Wenke et al., 2007, for details). No-go trials were signalled by colouring instructions after $2,700 \mathrm{~ms}$. In between the instruction of the identity task and the presentation of its imperative stimulus, a logically unrelated size task had to be performed. The size task did not require spatially defined responses and letter identity was not a response relevant attribute. Instead, two letters were presented alongside each other in a grid (see Fig. 1) $800 \mathrm{~ms}$ after instruction offset, and the task was to judge the position of the physically larger letter by pressing the space key once or twice. There were three types of size task displays that differed with respect to letter overlap and compatibility between size and identity (see Fig. 1). Task-overlapping letters appeared on half of the size task trials and were either spatially compatible or spatially incompatible across tasks. Spatially compatible size task displays were those in which the letters appeared at grid positions that spatially corresponded to the (response) locations assigned to these letters by letter task instructions (e.g., a small $\mathrm{N}$ on the left and a $\mathrm{K}$ in bigger print on the right). In incompatible trials, letter position in the size task display and instructed response location did not correspond (e.g., a small $\mathrm{K}$ on the left and a big $\mathrm{N}$ on the right side). Finally, in non-overlapping trials, other letters than those instructed for the identity task were presented as size task targets. These trials served as a neutral baseline condition.

When participants committed an error in the size task, a $200 \mathrm{~Hz}$ tone was presented during the $300 \mathrm{~ms}$ interval between their size task response and the onset of the identity task target. After the $300 \mathrm{~ms}$ interval a single letter appeared at a central screen position, and remained on the screen until response, or for a maximum of $1,000 \mathrm{~ms}$. This single letter served as a target for the letter identity task. On Go trials, participants responded according to the instructed mappings within the response window of $1,000 \mathrm{~ms}$. On Nogo trials they had to withhold responding to the letter and wait until the $1,000 \mathrm{~ms}$ had passed. When participants pressed the wrong key on Go trials, or when they failed to respond within the $1,000 \mathrm{~ms}$ response window, a $200 \mathrm{~Hz}$ tone was displayed during the $300 \mathrm{~ms}$ blank interval in the end of the trial. When they made a commission error on No-go trials they instead received written error feedback. The error message (mistake-responded though instruction had been coloured) remained on the screen for $2,000 \mathrm{~ms}$. The next trial started after a blank inter-trial-interval of $800 \mathrm{~ms}$.

For size task responses that required two successive key presses, we only considered the reaction time for the first key press because the inter-response intervals never reveal any effects with this paradigm (cf. Wenke et al., 2007).

Participants worked through two sessions of 400 trials with 16 blocks of 25 trials each. In all, 384 out of the 400 trials in each session were experimental (regular) trials. Assignment of specific letter pairs to the different overlap, compatibility, and size task response conditions were counterbalanced according to a Latin square (see Wenke et al., 2007, for details), with trial type (Go, No-go) as an additional factor in the counterbalancing scheme. Counterbalancing of letter pairs per condition across participants ensured (a) that one-third of the regular trials were No-go trials (128 total: 32 compatible, 64 neutral, 32 incompatible; equal frequencies for each combination of responses/to be avoided responses in the two tasks), and two-thirds of the trials were Go trials ( 256 total: 64 compatible, 128 neutral, 64 incompatible; equal frequencies for each combination of responses in the two tasks), and (b) that each participants saw the instructions for a specific letter pair only 4 times (twice in one session) during the entire experiment.

The remaining 16 trials in each session were catch trials (see Wenke et al., 2007). On catch trials, the letter task "target" was not amongst the instructed letters. Catch trials were added in order to ensure that participants encoded both S-R mappings in the instructions. As on No-go trials, participants were not supposed to respond to catch trial targets, and received the same written error message if they did.

Each session started with written instructions describing the procedure and the tasks, and three demo trials administered by the experimenter. Demonstration trials as well as practice trials featured digit instead of letter stimuli. The practice phase terminated when participants managed to complete a block of 24 practice trials with less 
than 11 errors (summed over both tasks), or after a maximum of three practice blocks. Experimental task instructions were then repeated with letter stimuli instead of digit stimuli, followed by four more practice trials containing letter stimuli, before the main part of the experiment started.

\section{Results}

Six participants were excluded from the analyses because they committed more than $20 \%$ errors across all conditions in the size task, the letter identity task, or both, leaving 24 participants in the analyses. This exclusion criterion was applied to ensure robust reaction time estimates (also see Wenke et al., 2007). Note that it is independent of performance differences between conditions.

For the analyses of reaction times (RTs), we only considered trials in which both the size task and the identity task responses were correct. Correct RTs that were more than 2.5 standard deviations away from an individual's mean per condition were discarded $(3.32 \%)$. For the remaining data, mean RTs for correct trials were determined for each participant and task as a function of crosstask compatibility (compatible, neutral, incompatible) and identity task trial type (Go vs. No-go; only applies to size task). As mentioned before, non-overlapping letter stimuli in the size task served as a neutral baseline against which we compared spatially compatible overlapping letters in order to assess costs associated with encountering the same letters in the two tasks. ${ }^{1}$

Individual error rates were calculated correspondingly, considering only responses with errors in one task. Trials in which errors were committed on both tasks were discarded $(0.39 \%)$. Table 1 shows the RTs and error data for each task and condition. It becomes evident from Table 1 that Go trial responses in the letter identity task were much slower in the incompatible than in the compatible and neutral conditions. More importantly, there was a small compatibility effect in size task responses on both, letter task Go trials and No-go trials. In addition, size task responses were faster on letter No-go than letter-go trials. Separate ANOVAS for the two tasks showed that all effects were reliable.

\footnotetext{
${ }^{1}$ We chose the difference between compatible and neutral trials instead of the overall difference between target-overlapping and non-overlapping trials because previous results (Wenke et al., 2007) showed that the compatibility effect in the size task was entirely due to incompatibility costs. Therefore, the compatible-neutral comparison seemed to be the purer measure of overlap costs that is less confounded with the compatibility effect.
}

Table 1 Mean reaction times (in ms) and errors (\%) for the size task and the letter identity task in incompatible, neutral (non-overlapping), and compatible letter task Go trials and letter task No-go trials in Exp. 1

\begin{tabular}{lrrrrr}
\hline $\begin{array}{l}\text { S(B) }-\mathrm{R}(\mathrm{A}) \\
\text { compatibility }\end{array}$ & \multicolumn{2}{l}{ Go trials } & & \multicolumn{2}{l}{ No-go trials } \\
\cline { 3 - 3 } \cline { 5 - 6 } & RT & $\begin{array}{l}\text { Percent } \\
\text { errors }\end{array}$ & & RT & $\begin{array}{l}\text { Percent } \\
\text { errors }\end{array}$ \\
\hline Size task & & & & & \\
Incompatible & 508.30 & 0.68 & & 450.32 & 1.37 \\
Neutral & 495.53 & 0.88 & & 444.43 & 1.17 \\
Compatible & 499.96 & 1.43 & & 439.40 & 1.37 \\
$\Delta$ & 8.34 & -0.75 & & 10.92 & 0.00 \\
Letter identity task & & & & \\
Incompatible & 544.13 & 7.88 & & - & 2.80 \\
Neutral & 512.28 & 4.59 & & - & 2.99 \\
Compatible & 509.27 & 4.00 & & - & 2.60 \\
$\Delta$ & 34.83 & 3.88 & & - & 0.20 \\
\hline
\end{tabular}

Go trial versus No-go trial refers to the identity task. The size task was always to be executed. The row labelled $\Delta$ depicts effect sizes of the compatibility effects (incompatible minus compatible)

\section{Size task}

Reaction times were submitted to a $2 \times 3$ ANOVA with trial type (letter task Go vs. No-go trials) and cross-task compatibility (incompatible, neutral, and compatible) as within-subjects factors. This analysis yielded a significant main effect of trial type, $F(1,23)=28.06, \quad P<0.01$, $\mathrm{MSE}=4103.48$, indicating that size task responses were faster in letter identity task No-go trials than in Go trials. Importantly, the main effect of compatibility was also significant, $F(2,46)=5.37, P<0.05$, MSE $=268.06$, whereas the interaction between compatibility and trial type was not, $F(2,46)=1.78, P>0.18$, MSE $=161.27$. Planned comparisons that tested the compatibility effects (i.e., the difference between incompatible and compatible trials) separately for each trial type revealed that both, the $8 \mathrm{~ms}$ compatibility effect in Go trials and the $11 \mathrm{~ms}$ effect in Nogo trials were reliable, $F(1,23)=5.01, P<0.05$, MSE = 333.06 , and $F(1,23)=10.43, \quad P<0.01, \quad \mathrm{MSE}=274.32$, respectively. Moreover, comparisons between the compatible and the neutral conditions for each trial type indicated that RTs for neutral responses did not differ from compatible responses for Go trials, $F(1,23)<1$, MSE $=629.42$, nor for No-go trials, $F(1,23)=2.64, P>0.11$, MSE $=229.39$, indicating that overlapping letter stimuli did not produce overlap costs (cf. Wenke et al., 2007).

Error rates in the size task were generally rather low, and neither differed between trial types, $F(1,23)=2.05$, $P>0.16, \mathrm{MSE}=1.62$, nor between compatibility conditions, $F(2,46)=2.79, \quad P>0.07, \quad \mathrm{MSE}=1.62$. The interaction 
between compatibility and trial type was not significant either, $F(2,46)=1.54, P>0.22$, MSE $=1.09$.

Additional analyses Although none of the effects reached significance in the error analysis, there was an almost significant trend for participants to make fewer errors on incompatible trials than on neutral and compatible trials, especially on Go trials. Therefore, we wanted to ensure that the pattern of results revealed by the RT analyses was not compromised by speed-accuracy tradeoffs. To this end, we conducted a $2 \times 3$ MANOVA that simultaneously considered RTs and errors as dependent variables. The resulting centroids for each effect in Exp. 1 are shown in panels a-c in the left column of Fig. 2. Consistent with the RT ANOVA, the MANOVA revealed significant main effects of trial type, $F(2,22)=14.09, P<0.01$, and compatibility,
$F(4,90)=4.54, P<0.01 \quad$ (all statistics based on Wilk's Lambda). The interaction between trial type and compatibility was again not significant, $F(4,90)=1.68, P>0.16$.

\section{Letter identity task}

The one-way ANOVA of RTs for letter identity Go trials with compatibility (compatible, neutral, incompatible) as a within-subjects factor showed that compatibility conditions differed from each other, $F(2,46)=20.25, P<0.01$, MSE = 442.09. Planned comparisons revealed that responses on compatible trials significantly differed from incompatible trials, $F(1,23)=26.09, P<0.01$, MSE $=1117.59$, whereas the difference between compatible and neutral trials was not significant, $F(1,23)<1$, MSE $=573.26$. a) trial-type Exp. 1

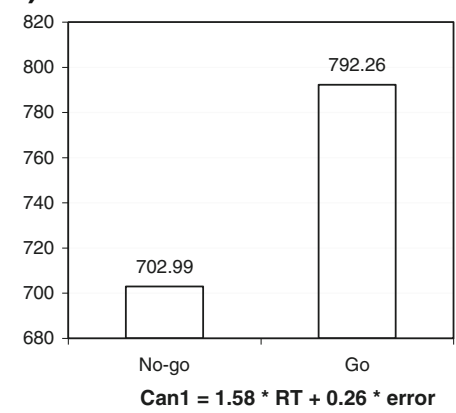

b) compatibility

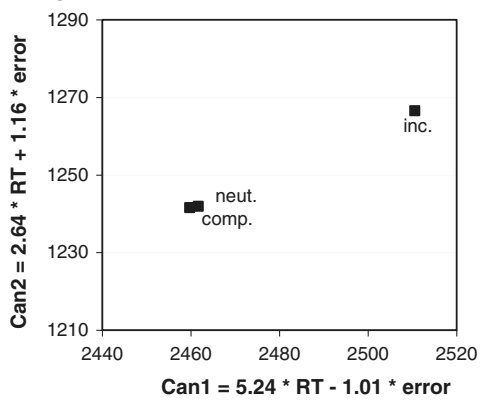

C) trial-type $X$ compatibility

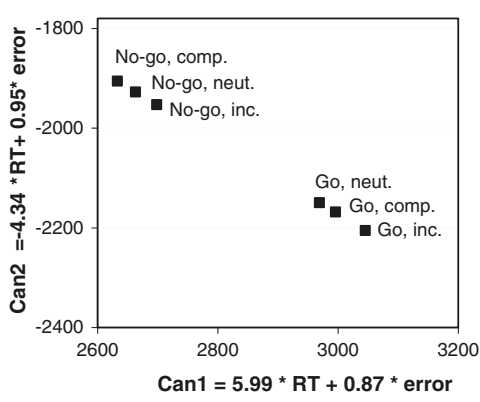

Exp. 2
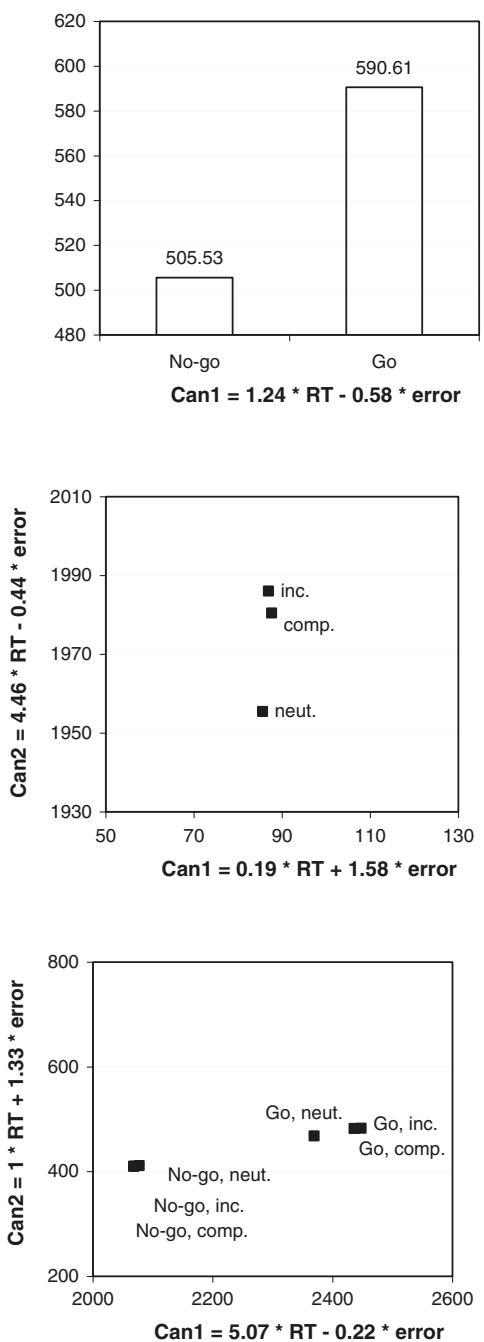

Exp. 3
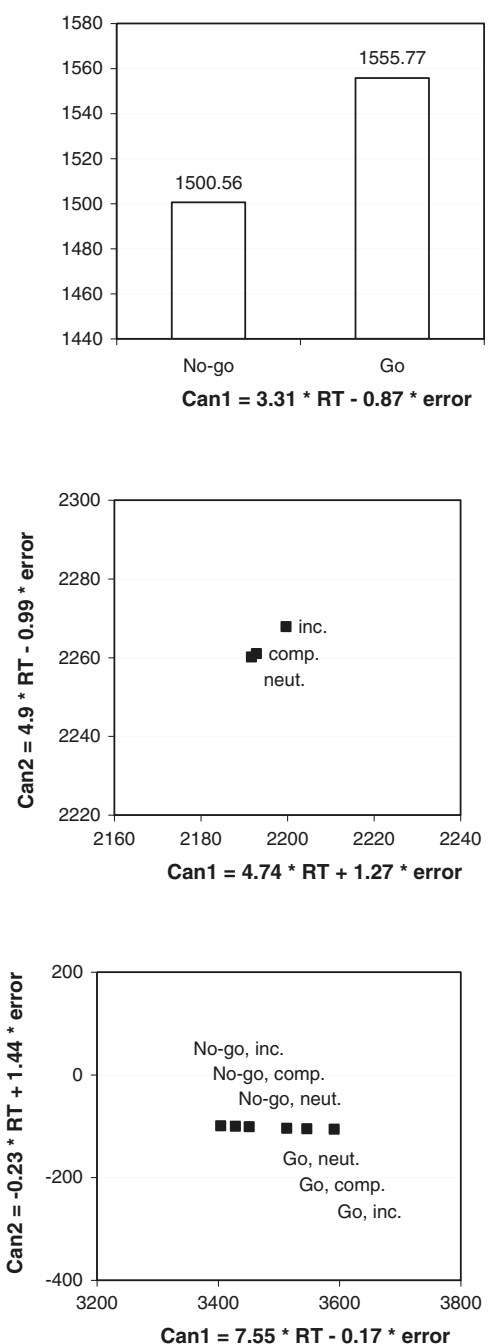

Fig. 2 MANOVA centroids for the main effects of trial type (a) and cross-task compatibility (b) as well as the interaction between trial type and compatibility (c), for Exps. 1, 2, and 3 (columns). Canl and
Can 2 refer to the first and second canonical functions determined by the MANOVA, respectively 
The corresponding one-way ANOVA of key errors in Go trials mirrored the RT results: the effect of compatibility was significant, $F(2,46)=24.4, \quad P<0.01, \quad \mathrm{MSE}=4.29$, indicating that participants committed more errors after incompatible size task displays than after neutral (nonoverlapping) or compatible displays.

Finally, commission errors on No-go trials did not differ amongst compatibility conditions, $F(2,46)<1$, MSE $=4.35$.

\section{Discussion}

The pattern of results was clear-cut with respect to both size task Go and No-go responses. Reactions were delayed when possible target letters of the succeeding identity task occupied spatial positions in the size task display that did not correspond to the instructed response locations. RTs of spatially corresponding responses were similar to RTs in neutral trials where the judged letters were different from the letters specified by instructions. That is, we obtained an interference-dominant pattern of results indicating RT costs for letters displayed at positions differing from the actually valid instructed $\mathrm{S}-\mathrm{R}$ mappings.

The compatibility effect in Go trials generally replicates the findings by Wenke et al. (2007; Exp. 1) and can be explained by instruction-induced $\mathrm{S}-\mathrm{R}$ binding. On this account (Wenke et al., 2007), representations of the instructed target features (i.e., letter identity) are linked to the instructed spatial features needed for later responding (e.g., $\mathrm{N}+\mathrm{LEFT}, \mathrm{K}+\mathrm{RIGHT}$ ) while reading the instructions, and the resulting links are implemented at a subverbal level for later use. When participants encounter the same letters in the interceding size task then letter identity, which is entirely irrelevant for the size task, automatically activates its associated spatial code. This in turn hampers stimulus identification (i.e., determining whether the bigger letter was on the left or the right) when the letters activate the "wrong" spatial codes, thereby slowing responses in the spatially incompatible condition. These instruction-induced S-R bindings are probably not of a verbal nature: first, the instructed mappings are completely irrelevant for performing the size task. Hence it is hard to see why participants would transform or translate a verbally represented rule during size task performance. Second, we found similar results as those observed here when the size task required judging the colour instead of the location of the bigger of two letters, and when participants held a tongue depressor in their mouths that supposedly blocked subvocal rehearsal (cf. Wenke et al., 2007, Exp. 2 and footnote 4). Hence we propose that quasi-perceptual or conceptual representations were integrated and bound at a subverbal level during some sort of cognitive simulation when situation models of the instructed situations were constructed (e.g., Barsalou, 1999; Glenberg \& Robertson, 2000).
The important new finding of the current Exp. 1 concerns the compatibility effect in No-go trials. Responses in incompatible size task trials were reliably delayed even when the instructed mappings for the letter identity task were rendered irrelevant by a No-go signal. Moreover, the compatibility effect in No-go trials was similar to the effect in Go trials, suggesting that participants started to implement instructions at a subverbal level when the mappings for the identity task were presented. When a No-go signal finally appeared in No-go trials, rule implementation had proceeded too far for successful abortion. As a consequence, the established S-R links that were no longer valid influenced subsequent behaviour in a reflex-like manner.

Although the impact of letter task instructions on size task performance did not differ between Go- and No-go trials, the significant main effect of trial type showed that size task responses were generally faster on No-go trials than on Go trials. Assuming that the instructed mappings were already implemented on a subverbal level when the size task came on, it is unlikely that the Go-trial slowing reflects additional working memory demands associated with subvocal rehearsal of the instructed verbal rules. Instead, Gotrial slowing might primarily reflect general dual task or mixing costs (e.g., Kray \& Lindenberger, 2000) related to keeping two tasks active and separate.

Finally, we also obtained an interference-dominant cross-task compatibility effect in the letter identity task. RTs were considerably delayed when the singular, centrally presented identity task target letter had occupied a position in the size task display that was spatially incompatible with the location of its instructed response.

The identity task effect can also be explained by bindinginduced conflict between spatial codes if one assumes that re-binding of letter identity and spatial position information occurs when responding to spatially incompatible size task displays. Accordingly, responding to size task displays integrates the encountered situational features (including letter identity and spatial position information) into some sort of S-R episode (Logan, Taylor, \& Etherton, 1999) or event file (Hommel, Müsseler, Aschersleben, \& Prinz, 2001). When one of the letters encountered in the size task then re-appears as identity task target it activates the other features in the event file. Activation of the "wrong" spatial code on incompatible trials delays selecting the appropriate (instructed) spatial response.

Viewed this way, the identity task results seem to be (yet) another demonstration of response-based binding and re-binding similar to those already reported in the literature (e.g., Hommel, 1998, 2007; Hommel \& Colzato, 2004; Waszak, Hommel, \& Allport, 2003), and are therefore less interesting for the current research question. In contrast, the size task results directly speak to the question of instruction implementation. They suggest that a top-down mechanism 
integrates relevant stimulus and response features solely on the basis of instructions.

The compatibility effect in No-go trials of Exp. 1 seems to favour the view that advance implementation of instructions is the default mode. However, in Exp. 1, No-go signals were infrequent and appeared late. Therefore, it is possible that the effect observed in Exp. 1 reflects a strategic decision to always implement instructions. That is, Exp. 1 leaves open to what extent participants can strategically control whether or not to implement instructions. This question was addressed in Exp. 2.

\section{Experiment 2}

Experiment 2 directly tested if participants strategically control whether they implement verbal instructions in advance or not. No-go signals appeared late during instruction presentation (after 2,700 ms, just like in Exp. 1). The results from Exp. 1 suggest that participants cannot successfully cancel advance implementation of instructions when No-go signals appear late, leading to automatic S-R activation. However, in Exp. 1, the proportion of No-go trials was much lower than the proportion of Go trials, so that participants presumably expected any given trial to be a Go trial. In Exp. 2, we reversed the ratio of Go and No-go trials such that No-go trials appeared twice as often as Go trials. The question of interest was whether participants could strategically decide not to implement instructions at a subverbal level when they expected the instructed mappings to be useless. If they do not implement the S-R instructions for the letter identity task, then there are no instruction-induced bindings which could become activated during size task performance. Consequently we should not expect an automatic influence of instructed mappings on size task performance.

Critically, such a strategic account predicts the effect to be absent in both, Go and No-go trials of the size task. The reason is simple: given that only very little time (300 ms) remains when the No-go signal does not appear at the expected time, participants cannot fully implement the instructed mappings on Go trials. As a result, no functional S-R bindings are established on Go trials either.

Methods

\section{Participants}

Thirty students from the city of Berlin (26 female, mean age $=21.8$ years, normal or corrected to normal vision) participated and received $€ 12$ or partial course credit.

\section{Design and procedure}

The design and the procedure of Exp. 2 were identical to Exp. 1, with the following exception: No-go signals appeared on $2 / 3$ instead of $1 / 3$ of the trials. Therefore, the total number of No-go trials was 256 (64 compatible, 128 neutral, 64 incompatible), whereas 128 trials were Go trials (32 compatible, 64 neutral, 32 incompatible).

\section{Results}

Four participants were excluded from the analyses because they committed more than $20 \%$ errors across all conditions in the size task, the letter identity task, or both. RTs and errors for the remaining 26 participants were processed as in the previous experiment. Trials with correct response RTs that were more than 2.5 standard deviations away from each participant's mean per condition were discarded $(3.25 \%)$, along with trials in which participants committed errors in both tasks $(0.41 \%)$. For the remaining data, individual mean RTs and percentages of errors were computed as a function of task, trial type, and compatibility condition. Table 2 summarises the resulting group means.

From inspection of the table, it becomes clear that letter identity task responses were again slower in the incompatible condition than in the compatible and neutral conditions. However, the differences between incompatible and compatible size task responses were negligible for both, identity task Go trials and No-go trials. Finally, size task responses were again faster on No-go than on Go trials. As in the previous experiments, we conducted separate ANOVAs for the size task and the letter identity task to test these effects.

Table 2 Mean reaction times (in $\mathrm{ms}$ ) and errors (\%) for the size task and the letter identity task in incompatible, neutral (non-overlapping), and compatible letter task Go trials and letter task No-go trials in Exp. 2

\begin{tabular}{|c|c|c|c|c|}
\hline \multirow{2}{*}{$\begin{array}{l}\mathrm{S}(\mathrm{B})-\mathrm{R}(\mathrm{A}) \\
\text { compatibility }\end{array}$} & \multicolumn{2}{|c|}{ Go trials } & \multicolumn{2}{|c|}{ No-go trials } \\
\hline & RT & $\begin{array}{l}\text { Percent } \\
\text { errors }\end{array}$ & RT & $\begin{array}{l}\text { Percent } \\
\text { errors }\end{array}$ \\
\hline \multicolumn{5}{|l|}{ Size task } \\
\hline Incompatible & 482.81 & 0.78 & 408.08 & 2.13 \\
\hline Neutral & 467.36 & 1.08 & 409.82 & 1.79 \\
\hline Compatible & 480.53 & 1.98 & 407.97 & 2.10 \\
\hline$\Delta$ & 2.28 & -1.20 & 0.11 & 0.03 \\
\hline \multicolumn{5}{|c|}{ Letter identity task } \\
\hline Incompatible & 516.99 & 12.56 & - & 1.23 \\
\hline Neutral & 494.39 & 7.99 & - & 0.98 \\
\hline Compatible & 493.44 & 6.73 & - & 1.23 \\
\hline$\Delta$ & 23.55 & 5.83 & - & 0.00 \\
\hline
\end{tabular}




\section{Size task}

The 2 (trial type) $\times 3$ (compatibility) within-subjects ANOVA of size task RTs revealed a significant main effect of trial type, $F(1,25)=66.37, P<0.01$, MSE $=2739.31$, with faster reactions for No-go than on Go trials. The main effect of compatibility was not significant, $F(2,50)=2.51$, $P>0.09, \mathrm{MSE}=277.53$, but the interaction between trial type and compatibility was reliable, $F(2,50)=5.8$, $P<0.01$. Planned comparisons that tested the compatibility effects separately for Go and No-go trials showed that the compatible and the incompatible conditions did neither differ for Go trials nor No-go trials (both $F \mathrm{~s}<1$ ). However, the difference between compatible and neutral trials approached significance in Go trials, $F(1,25)=4.09$, $P<0.06, \mathrm{MSE}=1103.9$, indicating that responses to nonoverlapping letter displays were faster. In No-go trials, the difference between compatible and neutral trials was not significant, $F(1,25)<1, \mathrm{MSE}=118.43$. Together, these comparisons suggest that the two-way interaction in the omnibus ANOVA was driven by differential overlap costs in Go and No-go trials.

The $2 \times 3$ ANOVA of size task errors yielded a significant main effect of trial type, $F(1,25)=15.99, P<0.01$, $\mathrm{MSE}=1.29$, again indicating that participants made more errors in No-go trials than in Go trials. The main effect of compatibility was significant, $F(2,50)=5.7, P<0.01$, MSE $=1.09$. The compatibility effect was qualified by a significant interaction between trial type and compatibility, $F(2,50)=3.23, P<0.05, \mathrm{MSE}=1.53$, indicating that participants made fewer errors in the incompatible Go trials than in compatible and neutral trials, $F(2,50)=7.02$, $P<0.01$, whereas the three compatibility conditions did not differ in No-go trials, $F(2,50)<1$.

Additional analyses In Exp. 2, size task RT and errors did not correspond well: while compatibility conditions primarily differed with respect to the neutral condition regarding RTs, the error data suggest that participants were actually better at performing incompatible than compatible (and neutral) trials. Moreover, responses in No-go trials were faster, but at the same time more error prone, than in Go trials. In order to control for potential speedaccuracy tradeoffs, we therefore again conducted a 2 (trial type) $\times 3$ (compatibility) MANOVA that simultaneously considered RTs and errors as dependent variables (see Fig. 2, for an overview of the resulting centroids). All effects in the MANOVA were significant: The main effect of trial type, $F(2,44)=37.8, P<0.01$, indicating lower canonical values for No-go trials than for Go trials, the main effect of compatibility, $F(4,98)=3.85, P<0.01$, as well as the interaction between trial type and compatibility, $F(2,98)=4.38, P<0.01$. Inspection of Fig. $2 b$, c suggests that the effect of compatibility was primarily due to a difference between neutral trials, on the one hand, and compatible and incompatible trials, on the other hand. These overlap costs were particularly pronounced for Go trials.

Finally, despite the well-known problems associated with comparing groups of different sizes, we directly compared the RTs of Exps. 1 and 2 in order to gain a clearer understanding of the differences between the two experiments. To this end, we submitted RTs to a 2 (experiment) $\times 2$ (trial type) $\times 2$ (compatibility) ANOVA. This ANOVA yielded significant main effects of trial type, $F(1,48)=76.06, \quad P<0.01$, and compatibility, $F(1,48)=$ $9.44, P<0.01$. Importantly, the interaction between experiment and compatibility was also significant, $F(1,48)=5.73$, $P<0.05$, indicating that the compatibility effect (across trial types) was larger in Exp. 1 than in Exp. 2. All other effects were not significant (all $F \mathrm{~s}<1, P>0.35$ ).

\section{Letter identity task}

The one-way ANOVA of RTs in letter identity Go trials with compatibility (compatible, neutral, incompatible) as a within-subjects factor revealed a significant difference between compatibility conditions, $F(2,50)=17.5, P<0.01$, $\mathrm{MSE}=264.03$. As in the previous experiments, planned comparisons showed that the main effect was primarily due to slower responses in incompatible trials than in compatible (and neutral) trials, $F(1,25)=27.93, P<0.01$, MSE = 516.46; compatible and neutral trials did not significantly differ from each other, $F(1,25)<1$, MSE = 453.27.

The overall percentage of key errors made in Go trials was higher than in Exp. 1, but the ANOVA of key errors confirmed that the pattern across compatibility conditions was the same: the effect of compatibility was significant, $F(2,50)=17.63, P<0.01, \mathrm{MSE}=13.87$, indicating that participants committed more errors after incompatible size task displays than after neutral (non-overlapping) or compatible displays.

In contrast, the percentage of commission errors in No-go trials was extremely low and did not differ across compatibility conditions, $F(2,50)<1, \mathrm{MSE}=0.83$.

\section{Discussion}

There was no indication of advance implementation of verbally instructed S-R mappings in Exp. 2, neither in No-go nor in Go trials: the overall $1.2 \mathrm{~ms}$ difference between compatible and incompatible size task responses was far from significant, and the $2.3 \mathrm{~ms}$ effect in Go trials did not differ from the $0.1 \mathrm{~ms}$ difference in No-go trials. Moreover, the compatibility effect in Exp. 2 was significantly smaller than in Exp. 1. 
Exp. 1 revealed that participants cannot interrupt verbal rule implementation when No-go signals appear very late during instruction presentation. Given that Exps. 1 and 2 differ only regarding the proportion of No-go trials, but not with respect to the timing of No-go signals, we therefore propose that the main difference between Exps. 1 and 2 concerns the strategic decision of whether or not to implement instructions in advance. On this view, participants in Exp. 1 expected a given trial to be a Go trial, and therefore decided to implement instructions when reading them more often than not. By contrast, participants in Exp. 2 presumably predicted No-go signals to appear on the majority of trials and therefore refrained from creating subverbal S-R links upon reading the instructions. As a consequence, taskoverlapping letters did not automatically activate their instructed responses when performing the size task.

This account seems generally consistent with Cohen, Jaudas, and Gollwitzer's (2008) claim that there may be more than one way to implement and act on intentions for future behaviour. Using a prospective memory task with varying numbers of prospective memory targets, Cohen et al. (2008) observed that monitoring for, and acting upon, only one prospective memory target did not incur any costs in a simultaneously performed lexical decision task. In contrast, two or more prospective memory targets did lead to costs. Based on these results, Cohen et al. (2008) concluded that participants used different encoding strategies when one versus many targets were instructed. Specifically, they suggested that participants form spontaneous if-then plans in the one-target condition that lead to automatic rule activation once the target is encountered. In contrast, "with more complex prospective memory instructions [...] participants must employ some alternative strategy" (Cohen et al., 2008, p. 155).

What could be such an "alternative strategy" for performing the letter identity task in our Exp. 2? Possibly, participants maintained a purely verbal representation of the instructed rules in verbal working memory (i.e., the articulatory loop) and rehearsed them subvocally (see Baddeley, 2003 , for a review) until the identity task target was presented. According to this account, they did not implement the instructed mappings on a sub-linguistic level while reading the instructions. Instead they retrieved the verbal rule from memory and translated it into behaviour once they encountered the target-perhaps assisted by verbal self-instructions (e.g., "There is an $\mathrm{N}$, that means right"; Baddeley, Chincotta, \& Adlam, 2001; Goschke, 2000; Kray, Eenshuistra, Kerstner, Weidema, \& Hommel, 2006, Luria, 1959; Miyake, Emerson, Padilla, \& Ahn, 2004). In line with this view, participants responded substantially more often with the wrong key (9\% on average) in letter task Go trials in Exp. 2 than in Exps. 1 and 3 (both about $5 \%$ ), whereas they committed notably fewer No-go errors.
The high Go trial error rate suggests that participants often failed to retrieve the correct verbal S-R rule when the trial was a Go trial.

However, if participants had exclusively used a memory retrieval and ad hoc implementation strategy at target presentation, one might also have expected generally slower letter task responses in Exp. 2 than in Exp. 1. This was not the case, however. One explanation for the failure to obtain reliable RT differences between experiments is that participants just chose the response that corresponded to the conjunction between letter identity and (letter) position encountered in the size task when retrieval of the instructed mapping was difficult. The huge compatibility effect in the letter task error data of Exp. 2 (compared to Exps. 1 and 3) supports this view.

Another noteworthy result in Exp. 2 concerns the overlap costs in the size task. The RT ANOVA and the MANOVA showed that size task responses were considerably impaired in target overlapping compared to neutral trials. The interaction between trial type and compatibility furthermore revealed that these overlap costs were particularly pronounced in the generally slower Go trials. Assuming that participants in Exp. 2 did not fully implement the instructed mappings while reading them, the overlap costs observed in Exp. 2 could reflect additional challenges related to actively maintaining verbal representations in verbal working memory when a trial turned out to be a Go-trial. In this view, overlapping size task targets triggered partial retrieval of the instructed rules, the implementation of which then had to be further postponed.

\section{Experiment 3}

The results from Exps. 1 and 2 suggest that participants can use the probability of No-go signals for deciding whether or not to implement instructions in advance. In Exp. 3 we investigated whether participants can abort implementation of verbal rules when they appear early enough and when there is enough time to interrupt implementation.

Exp. 3 was similar to Exp. 1 in that No-go signals only appeared in one-third of the trials. Unlike Exp. 1, however, the colour change of instructions signalling No-go trials already occurred early after instruction onset (see Fig. 1). This manipulation should give participants ample time to interrupt instruction implementation in No-go trials, and leave enough time to fully implement instructions in Go trials (Hourihan \& Taylor, 2006). If participants can adaptively encode or interrupt, this should be reflected in a dissociation of size task performance in Go trials and in No-go trials. That is, we should observe a cross-task compatibility effect in Go trials, but not in No-go trials. 
Methods

\section{Participants}

Thirty-eight students from the city of Berlin (23 female, mean age $=24.4$ years, normal or corrected to normal vision) participated and received $€ 12$ or partial course credit.

\section{Design and procedure}

The design and the procedure were identical to Exp. 1, except for the timing of No-go signals: On No-go trials, the mapping instructions for the letter identity task turned red after $400 \mathrm{~ms}$, and stayed red for the remaining 2,600 ms of instruction presentation.

\section{Results}

Six participants were excluded from the analyses according to the same criteria as in Exps. 1 and 2, leaving 32 participants for the analyses. As in Exp. 1, trials with correct response RTs that were more than 2.5 standard deviations away from a participant's mean per condition were discarded $(3.33 \%)$, along with trials in which participants committed errors in both tasks $(0.33 \%)$. For the remaining data, individual mean RTs and percentages of errors were computed as in the other experiments. Table 3 shows the means for each task, trial type, and compatibility condition.

The table indicates that letter identity task responses were again slower in the incompatible condition than in the compatible and neutral conditions. As in the previous experiments, size task responses were faster for No-go than Go trials. There also was a tendency towards a compatibil-

Table 3 Mean reaction times (in $\mathrm{ms}$ ) and errors (\%) for the size task and the letter identity task in incompatible, neutral (non-overlapping), and compatible letter task Go trials and letter task No-go trials in Exp. 3

\begin{tabular}{|c|c|c|c|c|}
\hline \multirow{2}{*}{$\begin{array}{l}\mathrm{S}(\mathrm{B})-\mathrm{R}(\mathrm{A}) \\
\text { compatibility }\end{array}$} & \multicolumn{2}{|c|}{ Go trials } & \multicolumn{2}{|c|}{ No-go trials } \\
\hline & RT & $\begin{array}{l}\text { Percent } \\
\text { errors }\end{array}$ & RT & $\begin{array}{l}\text { Percent } \\
\text { errors }\end{array}$ \\
\hline \multicolumn{5}{|l|}{ Size task } \\
\hline Incompatible & 475.74 & 2.27 & 450.97 & 3.03 \\
\hline Neutral & 466.28 & 1.99 & 457.19 & 2.83 \\
\hline Compatible & 469.74 & 2.20 & 454.16 & 2.88 \\
\hline$\Delta$ & 6.00 & 0.07 & -3.19 & 0.12 \\
\hline \multicolumn{5}{|c|}{ Letter identity task } \\
\hline Incompatible & 502.75 & 7.15 & - & 2.25 \\
\hline Neutral & 488.03 & 4.41 & - & 0.83 \\
\hline Compatible & 492.00 & 3.91 & - & 1.95 \\
\hline$\Delta$ & 10.75 & 3.24 & - & 0.30 \\
\hline
\end{tabular}

ity effect in size task RTs, but only for Go trials. In No-go trials, letter task instructions did not influence size task performance-at least not in the usual direction. These observations were tested in separate ANOVAs for each task.

\section{Size task}

Reaction times were submitted to a 2 (trial type) $\times 3$ (compatibility) within-subjects ANOVA. This analysis revealed a significant main effect of trial type, $F(1,31)=23.27$, $P<0.01, \mathrm{MSE}=560.15$, indicating that size task responses were faster for letter identity task No-go trials than Go trials. The main effect of compatibility was not significant, $F(2,62)<1, \operatorname{MSE}=181.10$, but the interaction between trial type and compatibility was, $F(2,62)=6.66, P<0.01$, MSE $=149.16$. Planned comparisons testing the compatibility effects (i.e., RTs in incompatible vs. compatible trials) separately for Go and No-go trials showed that the $6 \mathrm{~ms}$ difference in Go trials approached significance, $F(1,31)=3.96, P<0.06, \mathrm{MSE}=291.97$, whereas the effect in No-go trials did not, $F(1,31)<1$, MSE $=438.31$. Additional comparisons of the compatible and the neutral conditions indicated that RTs for neutral responses did not differ from compatible responses, neither for Go trials, $F(1,31)=1.4, P>0.24, \mathrm{MSE}=271.79$, nor for No-go trials, $F(1,31)<1$, MSE $=299.77$.

Error rates in the size task significantly differed between trial types, $F(1,31)=9.53, P<0.01$, MSE $=2.92$, indicating that participants made more errors on No-go trials than on Go trials. Neither the main effect of compatibility nor the interaction between trial type and compatibility were significant (both $F \mathrm{~s}<1, P>0.6$ ).

Additional analyses As in Exp. 1, we additionally conducted a 2 (trial type) $\times 3$ (compatibility) MANOVA with $\mathrm{RT}$ and errors as dependent variables in order to back up the RT results. The MANOVA results were generally consistent with the RT ANOVA: the main effect of trial type was significant, $F(2,30)=17.22, P<0.01$, reflecting the fact that the canonical values were lower for No-go than Go trials (see Fig. 2a). The only other significant effect was the interaction between trial type and compatibility, $F(4,122)=3.17, P<0.05$, again suggesting that there was a difference between compatibility conditions in Go but not in No-go trials (see Fig. 2c). The main effect of compatibility was not significant, $F(4,122)<1$.

Finally, we directly compared Exps. 1 and 3, conducting a 2 (experiment) $\times 2$ (trial type) $\times 2$ (compatible, incompatible) mixed factors ANOVA. This ANOVA yielded significant main effects of trial type, $F(1,54)=50.75$, $P<0.01, \mathrm{MSE}=1705.59$, and compatibility, $F(1,54)=9.81$, $P<0.01, \mathrm{MSE}=170.48$. The RT difference between Go and No-go trials was larger in Exp. 1 than in Exp. 3, as indicated by a significant interaction between experiment and 
trial type, $F(1,54)=12.29, P<0.01$. Furthermore, the compatibility main effect was qualified by the two-way interaction between experiment and compatibility, $F(1,54)=5.43$, $P<0.05$, indicating that the compatibility effect was larger in Exp. 1 than in Exp. 3. The three-way interaction between experiment, trial type, and compatibility missed significance, $F(1,54)=2.82, P=0.09$, MSE $=168.48$. However, post hoc comparisons suggest that the compatibility effects in Exps. 1 and 3 primarily differed in No-go trials: They revealed a highly significant compatibility effect across experiments in Go trials, $F(1,54)=9.12, P<0.01$, that did not interact with experiment, $F(1,54)<1$. In comparison, the contrast testing the main effect of compatibility in Nogo trials was not significant, $F(1,54)=2.23, P>0.14$, whereas the comparison testing the interaction between compatibility and experiment was significant, $F(1,54)=$ 7.4, $P<0.01$.

\section{Letter identity task}

The one-way ANOVA of RTs for letter identity Go trials with compatibility (compatible, neutral, incompatible) as a factor showed that compatibility conditions differed significantly from each other, $F(2,62)=10.01, P<0.01$, MSE = 185.30. Planned comparisons revealed that responses in compatible trials differed significantly from incompatible trials, $F(1,31)=9.56, P<0.01, \mathrm{MSE}=386.32$, whereas the difference between compatible and neutral trials was not significant, $F(1,31)=1.94, P>0.17, \mathrm{MSE}=260.70$.

The corresponding one-way ANOVA of key errors for Go trials mirrored the RT results: The effect of compatibility was significant, $F(2,62)=18.91, P<0.01, \mathrm{MSE}=5.17$, indicating that participants committed more errors after incompatible size task displays than neutral (non-overlapping) or compatible displays.

Unexpectedly, the percentage of commission errors for No-go trials also differed amongst compatibility conditions, $F(2,62)=6.74, P<0.01$, MSE $=2.65$, indicating that participants made fewer commission errors following non-overlapping (neutral) size task stimuli than following overlapping (compatible and incompatible) displays.

\section{Discussion}

Size task responses in Exp. 3 showed a small compatibility effect in Go trials but not in No-go trials, as indicated by the interaction between trial type and compatibility (in the RT ANOVA and the MANOVA). In addition, the compatibility effects in Exps. 1 and 3 primarily differed with respect to No-go trials. Together, these findings indicate that participants had some control over verbal rule implementation even on a trial-to-trial basis. However, several aspects of our findings suggest that trial-to-trial adaptation to task demands was imperfect and error prone. On the one hand, the difference between incompatible and compatible size task responses in Go trials was numerically even smaller than in the first experiment, and was only marginally significant when tested alone, suggesting that participants did not always start to implement instructions when they were presented. On the other hand, the RT difference between Go and No-go trials (i.e., the trial type effect) was smaller in Exp. 3 than in Exp. 1. In addition, participants made more commission errors on No-go trials of the identity task in Exp. 3 than in Exp. 1, especially when the identity task target was preceded by a letteroverlapping size task display (i.e., in the compatible and incompatible conditions). The latter findings indicate that not implementing instructions on No-go trials was imperfect, too, and that responding to instructed, but "invalid" letters during size task performance re-introduced some sort of "Go trial set" that led participants to respond to No-go targets.

One possible explanation for these results is that participants in Exp. 3 delayed instruction implementation until the expected time of a No-go signal. That is, they only started to prepare when no No-go signal appeared early during instruction presentation, still allowing enough time for at least partial implementation. This in turn resulted in (partial) automatic retrieval of the implemented mappings during size task performance. Conversely, participants did not begin to implement instructions if a No-go signal appeared.

However, such a "wait and see" strategy seems quite maladaptive in situations that primarily consist of Go trials. In particular, it is unlikely that participants decided to wait although they expected a given trial to be a Go trial. This would imply an endogenous switch from not preparing to late preparation on the majority of trials. In addition, such a strategy cannot explain those results that suggest imperfect cancellation of implementation on No-go trials (see above; also see "General discussion"). We therefore favour an interruption explanation of our Exp. 3 data. Accordingly, participants started to implement instructions as soon as the mappings were presented on a substantial proportion of trials. Unlike Exp. 1, however, they could use the early No-go signals to interrupt implementation. Such an explanation seems in line with findings by Hourihan and Taylor (2006) who investigated intentional control of directed forgetting. They combined an item-method directed-forgetting task with a stop-signal task, varying the onset of stop signals (forget cues) during item presentation. Similar to our Exp. 1, participants could not successfully interrupt item encoding when the forget cues arrived late, as indicated by comparable recall of to be remembered and to be forgotten items. In contrast, they found a directed-forgetting effect with early forget cues. The authors interpreted their findings as indicating 
that early cues biased a race between (default) deep encoding and forgetting.

In sum, the results from Exp. 3 can be explained by a race process between externally triggered encoding of instructed mappings and a "forget" cue. When No-go signals appear early, participants can use this information to interrupt implementation before functional links between the instructed stimulus and response features are established. This kind of flexible adaptation seems to be effortful and error prone (also see Philipp, Joliceur, Falkenstein, \& Koch, 2007).

\section{General discussion}

In three experiments we investigated temporal and strategic factors affecting whether participants implement verbal task instructions in a way that allows future task performance in a prepared-reflex like manner. The cross-task compatibility effect in the unrelated size task immediately following binary choice letter task instructions on each trial (Wenke et al., 2007) served as a measure of automatic activation of the instructed mappings, and hence, as an indicator of advance implementation of instructions. Exp. 1 showed that the size task compatibility effect was preserved in No-go trials when No-go signals appeared infrequently and late during instruction presentation. Compatibility effects were absent in both, size task responses in No-go trials and in Go trials, when No-go signals instead appeared frequently and late in Exp. 2. Finally, a small size task compatibility effect was observed on Go trials, but not on No-go trials when No-go signals appeared infrequently and early instead of late in Exp. 3.

The compatibility effect in the No-go trials of Exp. 1 supports a strong automaticity view. It extends previous findings indicating that verbal instructions can suffice to create bindings between the instructed stimulus and response features that become automatically activated whenever the specified stimulus conditions are met (CohenKdoshay \& Meiran, 2007; De Houwer et al., 2005, Wenke et al., 2007). Our findings show that merely instructed mappings become activated even when they are rendered useless by No-go signals, and hence seem to behave much like well-practiced S-R mappings. Other findings suggest, however, that merely instructed and already practiced mappings might functionally differ in some regards. For example, the only two studies so far that directly compared merely instructed and already practiced mappings report functional (Waszak et al., 2008) as well as neuroanatomical (Brass et al., 2009) dissociations between the two types of mappings in a task switching paradigm that assessed crosstalk elicited by the two types of mappings. Waszak et al. (2008) as well as Brass et al., 2009 concluded that practice establishes links at a direct, sensorimotor level, whereas merely instructed mappings might be represented at a more abstract task set level.

Consistent with this interpretation, Cohen-Kdoshay and Meiran (2007; Exp. 4) demonstrated that automatic activation of merely instructed S-R rules depends on working memory load. In Eriksen flanker experiments with merely instructed (not yet practiced) flanker stimuli serving as flankers, Cohen-Kdoshay and Meiran (2007) observed substantial flanker compatibility effects (i.e., faster responses when the flankers were mapped to the same response as the target, compared to flankers assigned to another response) only when participants could focus on the flanker task. The effect disappeared when memory load was increased. In contrast, results by Hommel and Eglau (2002) suggest that working memory load does not affect automatic S-R translation of practiced mappings. Hommel and Eglau (2002) observed a robust backward compatibility effect in dual task experiments with overlapping response codes from task 2 to task 1 that was independent of working memory load. Therefore, they concluded that practice presumably establishes direct and permanent $\mathrm{S}-\mathrm{R}$ associations that do not (or no longer) depend on temporary links held in working memory.

Taken together, these findings suggest that instructions can be used to create temporary sub-linguistic control structures or task sets in working memory, whereas practice might lead to direct S-R links at a sensorimotor level. The size task compatibility effect in No-go trials of Exp. 1 indicates that such instruction-induced temporary S-R links (Hommel \& Eglau, 2002) can be quite tenacious. Participants could not effectively "delete" the instructed S-R mappings from their working memory representation when No-go signals rendered them useless late during instruction presentation.

While Exp. 1 indicates that participants have little control over already implemented mappings, the results from Exps. 2 and 3 suggest that participants can exert some control over the implementation process itself, thus generally confirming the claims based on the prepared reflex metaphor. The results from Exp. 2 suggest that instruction implementation is under strategic control. Although No-go signals were presented as late as in Exp. 1, the compatibility effect in the size task was absent in both No-go trials and Go trials. In addition, the error rate in letter task Go trials was higher than in Exp. 1, whereas the rate of commission errors on No-go trials was lower. Together, these findings indicate that participants adopted a "wait and see" strategy (Hourihan \& Taylor, 2006) when noticing that a given trial was likely to be a No-go trial. While it may not be particularly surprising that participants can strategically adapt the amount of task preparation to frequency biases given previous findings with practiced tasks (Kleinsorge \& Gajewski, 
2004; Low \& Miller, 1999; Ramautar, Kok, \& Ridderinkhof, 2004), it is interesting to see that participants also decide to withhold instruction implementation when the source is unreliable. Whereas deciding not to prepare a practiced mapping presumably implies de-activating or not activating already implemented mappings, applying the same strategy to merely instructed mappings requires that instructions can be used to guide future behaviour in a different manner than by implementing them in advance. We propose that such an alternative route of instruction following might involve maintenance and (subvocal) rehearsal of the instructed verbal rules in verbal working memory, followed by ad hoc transformation of rules once the imperative stimulus appears. The high error rate in letter task Go trials indicates that such an alternative strategy may be less efficient than implementing instructions in advance. The large compatibility effect in Go trial errors of the letter task furthermore suggests that participants were strongly influenced by the event files (Hommel et al., 2001) created during size task performance when retrieving and translating the instructed rules during letter task performance.

Finally, the presence of a compatibility effect in size task performance in Go trials of Exp. 3, and its absence in Nogo trials, indicate that participants can flexibly interrupt instruction implementation on a trial-to-trial basis when No-go signals appear early. We suggest that the combined results from Exps. 1 and 3 can best be explained by a race account (Hourihan \& Taylor, 2006) according to which early, but not late, "forget cues" bias a race between deep encoding of instructions and instruction cancellation. Our findings leave open, however, whether it is possible to intentionally "undo" already implemented mappings, provided there is enough time. Future experiments that address this question will ideally include a low number of late Nogo signals like in Exp. 1, but will allow additional time between the No-go signal and instruction offset. ${ }^{2}$

In sum, the current experiments investigated how verbal instructions are used to control future behaviour, an ability that has been regarded as a cornerstone of cognitive flexibility and voluntary behaviour (Luria, 1961; Tolman, 1948). Our findings suggest that there may be at least two different ways how instructions come to control behaviour, and that humans can strategically decide between them. The results from Exps. 1 and 3 indicate that the preferred strategy with a reliable source or instructor is to implement instructions on a subverbal level even before the instructed stimulus conditions are encountered for the first time, allowing automatic stimulus-response activation from trial one. While advance implementation of instructions efficiently delegates control to the environment and thereby

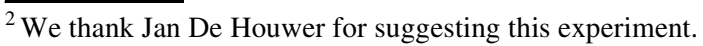

frees resources for other activities (Gollwitzer, 1999), it also bears risks: instructions that are rendered invalid late during encoding cannot easily be deleted from the already implemented control structure. As a result, the implemented, but entirely useless mappings automatically influence ongoing behaviour. An alternative route to instruction following involves postponing verbal rule transformation until the instructed stimulus condition is met. Such a "wait and see" strategy adopted by participants when No-go signals are frequent (Exp. 2) appears to be an efficient strategic adaptation to situations where the source providing the instructions is considered unreliable.

Acknowledgments We thank Hendrik Lohse, Stefanie Schmidt, Maria Eichhorn, and Jens Nachtwei for collecting the data, and Jan De Houwer as well as an anonymous reviewer for helpful comments regarding an earlier version of the manuscript.

Open Access This article is distributed under the terms of the Creative Commons Attribution Noncommercial License which permits any noncommercial use, distribution, and reproduction in any medium, provided the original author(s) and source are credited.

\section{References}

Baddeley, A. (2003). Working memory: Looking back and looking forward. Nature Reviews Neuroscience, 4, 829-839.

Baddeley, A., Chincotta, D., \& Adlam, A. (2001). Working memory and the control of action: Evidence from task switching. Journal of Experimental Psychology: General, 130, 641-657.

Barsalou, L. W. (1999). Perceptual symbol systems. Behavioral and Brain Sciences, 22, 577-660.

Brass, M., Wenke, D., Spengler, S., \& Waszak, F. (2009). Neural correlates of overcoming interference from instructed and implemented stimulus-response associations. Journal of Neuroscience, $29,1766-1772$.

Cohen, A., Jaudas, A., \& Gollwitzer, P. M. (2008). Number of cues influences the cost of remembering to remember. Memory \& Cognition, 36, 149-156.

Cohen-Kdoshay, O., \& Meiran, N. (2007). The representation of instructions in working memory leads to autonomous response activation: Evidence from the first trials in the flanker paradigm. The Quarterly Journal of Experimental Psychology, 60, 11401154.

Cohen-Kdoshay, O., \& Meiran, N. (2009). The representation of instructions operates like a prepared reflex: Flanker compatibility effects found in the first trial following S-R instructions. Experimental Psychology, 56, 128-133.

Colgan, D. M. (1970). Effects of instructions on the skin resistance response. Journal of Experimental Psychology, 86, 108-112.

Cook, S. W., \& Harris, R. E. (1937). The verbal conditioning of the galvanic skin reflex. Journal of Experimental Psychology, 21, 202-210.

De Houwer, J., Beckers, T., Vandorpe, S., \& Custers, R. (2005). Further evidence for the role of mode-independent short-term associations in spatial Simon effects. Perception \& Psychophysics, 67, 659-666.

Exner, S. (1879). Physiologie der Grosshirnrinde. In L. Hermann (Ed.), Handbuch der Physiologie (Vol. 2, pp. 189-350). Leipzig, Germany: Vogel. 
Glenberg, A. M., \& Robertson, D. A. (2000). Symbol grounding and meaning: A comparison of high-dimensional and embodied theories of meaning. Journal of Memory and Language, 43, 379-401.

Gollwitzer, P. (1999). Implementation intentions: Strong effects of simple plans. American Psychologist, 54, 493-503.

Goschke, T. (2000). Intentional reconfiguration and voluntary persistence in task set switching. In S. Monsell \& J. Driver (Eds.), Attention and Performance XVIII: Control of cognitive processes (pp. 331-355). Cambridge, MA: MIT Press.

Hommel, B. (1998). Event files: Evidence for automatic integration of stimulus-response episodes. Visual Cognition, 5, 183-216.

Hommel, B. (2000). The prepared reflex: Automaticity and control in stimulus-response translation. In S. Monsell \& J. Driver (Eds.), Attention and performance XVIII: Control of cognitive processes (pp. 247-273). Cambridge: MIT Press.

Hommel, B. (2007). Feature integration across perception and action: Event files affect response choice. Psychological Research, 71, $42-63$.

Hommel, B., \& Colzato, L. (2004). Visual attention and the temporal dynamics of feature integration. Visual Cognition, 11, 483-521.

Hommel, B., \& Eglau, B. (2002). Control of stimulus-response translation in dual-task performance. Psychological Research, 66, 260-273.

Hommel, B., Müsseler, J., Aschersleben, G., \& Prinz, W. (2001). The theory of event coding (TEC): A framework for perception and action. Behavioral and Brain Sciences, 24, 849-878.

Hourihan, K. L., \& Taylor, T. L. (2006). Cease remembering: Control processes in directed forgetting. Journal of Experimental Psychology: Human Perception and Performance, 32, 1354-1365.

Kleinsorge, T., \& Gajewski, P. D. (2004). Preparation for a forthcoming task is sufficient to produce subsequent shift costs. Psychonomic Bulletin \& Review, 11, 302-306.

Kray, J., Eenshuistra, R., Kerstner, H., Weidema, M., \& Hommel, B. (2006). Language and action control: The acquisition of action goals in early childhood. Psychological Science, 17, 737-741.

Kray, J., \& Lindenberger, U. (2000). Adult age differences in task switching. Psychology and Aging, 15, 126-147.
Kunde, W., Kiesel, A., \& Hoffmann, J. (2003). Conscious control over the content of unconscious cognition. Cognition, 88, 223-242.

Logan, G. D., Taylor, S. E., \& Etherton, J. L. (1999). Attention and automaticity: Toward a theoretical integration. Psychological Research, 62, 165-181.

Low, K. A., \& Miller, J. (1999). The usefulness of partial information: Effects of go probability in the choice/nogo task. Psychophysiology, 36, 288-297.

Luria, A. R. (1959). The directive function of speech in development and dissolution. Word, 15, 341-352.

Luria, A. R. (1961). The role of speech in the regulation of normal and abnormal behavior. New York: Liveright Publishing Corporation.

Miyake, A., Emerson, M. J., Padilla, F., \& Ahn, J. C. (2004). Inner speech as a retrieval aid for task goals: The effects of cue type and articulatory suppression in the random task cuing paradigm. Acta Psychologica, 115, 123-142.

Philipp, A. M., Jolicoeur, P., Falkenstein, M., \& Koch, I. (2007). Response selection and response execution in task switching: Evidence from a go-signal paradigm. Journal of Experimental Psychology: Learning, Memory, and Cognition, 33, 1062-1075.

Ramautar, J. R., Kok, A., \& Ridderinkhof, K. R. (2004). Effects of stop-signal probability in the stop-signal paradigm: The N2/P3 complex further validated. Brain and Cognition, 56, 234-252.

Tolman, E. C. (1948). Cognitive maps in rats and men. Psychological Review, 55, 189-208.

Waszak, F., Hommel, B., \& Allport, A. (2003). Task-switching and long-term priming: Role of episodic stimulus-task bindings in task-shift costs. Cognitive Psychology, 46, 361-413.

Waszak, F., Wenke, D., \& Brass, M. (2008). Cross-talk of instructed and applied arbitrary visuomotor mappings. Acta Psychologica, 127, 30-35.

Wenke, D., Gaschler, R., \& Nattkemper, D. (2007). Instructioninduced feature binding. Psychological Research, 71, 92-106.

Woodworth, R. S. (1938). Experimental psychology. New York: Holt, Rinehart and Winston. 